\title{
6. COMMISSION DES TÉLÉGRAMMES ASTRONOMIQUES
}

PrÉSIDENT: M. H. SPENCER Jones, Astronomer Royal, Royal Observatory, Greenwich, London, England.

Directeur du Bureau: M. E. Strömgren.

Membres: MM. B. H. Dawson, J. Jackson, H. Shapley.

From April 6, I935 (date of the last report) up to the present date (Feb. 26, I938) the Bureau has distributed 723 telegrams and published 174 circulars (Nos. 535$708)$.

The Bureau has again been supported by a great many colleagues and institutions.

As heretofore, the leading principle in our work has been as far as possible to procure provisional data for the continuous observation of new objects. The telegraphic service has been used when necessary to safeguard new discoveries, while in all cases possible the circulars have been depended on. Thus, as before, in dubious cases we have, to begin with, sent a telegram to a few correspondents only, asking for control. In cases of rediscovery of periodic comets and in other cases, when there was no risk of losing the object, we have distributed the announcement through the circulars.

Summing up it can be said that the endeavour has been made to reduce the costs of the telegraphic service as much as possible without the risk of losing new objects. With a few correspondents we have had the arrangement that we communicate all telegrams to them, and of course we are ready to arrange this with any correspondent who might wish it.

The difficulties arising from different systems of counting time and equinox have been completely got rid of. The rules for uniformity in the dates used in ephemerides proposed at the Leiden meeting have been accepted by our correspondents.

In the work of the Bureau I have been effectively assisted by Miss Vinter Hansen, Messrs Möller, Thernöe and Hjerting and-in the period April I935 till Sept. I936by Prof. B. Strömgren. In the correspondence and in general work $\mathrm{I}$ have been to a great extent assisted by Miss Mackeprang.

\section{Actual List of Subscribers to Telegrams and Circulars}

Harvard College Observatory, Cambridge, Mass., U.S.A.

Observatory, Capetown, South Africa.

Observatory, Melbourne, South Yarra, Australia.

Observatory, Edinburgh, Scotland.

Observatory, Greenwich, England.

Norman Lockyer Observatory, Sidmouth, England.

Isidro Polit, Servei d'Astronomia de la Generalitat de Catalunya, Barcelona, Spain.

Observatorio astronomico, Madrid, Spain.

Observatorio Marina, San Fernando, Spain.
Observatoire, Besançon, France.

Observatoire, Floirac-Bordeaux, France.

Observatoire, Marseille, France.

Observatoire, Nice, France.

Observatoire, Paris, France.

Observatoire, Strasbourg, France.

Observatoire, Alger, North Africa.

Sterrewacht, Leiden, Holland.

Osservatorio Capodimonte, Napoli, Italy.

Osservatorio, Milano, Italy.

Osservatorio, Padova, Italy.

Osservatorio, Pinotorinese, Italy.

Observatoire, Bucarest, Roumania.

Observatoriet, Saltsjöbaden-Stockholm, Sweden. 
Observatoire, Genève, Switzerland.

Observatory, Helwan, Egypt.

Observatoire, Vladivostock, Russia.

Observatoire, Uccle, Belgium.

Observatory, Tokyo, Japan.

Observatoire d'Astronomie physique, Meudon, France.

Royal Astronomical Society, London, England.

Osservatorio, Monte Mario, Roma, Italy.

Observatoire, Athènes, Greece.
Osservatorio Astronomico, Trieste, Italy. Institut Astronomique, Leningrad, Russia. Obserwatorium, Poznań, Poland.

Observatoriet, Upsala, Sweden.

P. Emanuelli, Roma, Italy.

Science Service, Washington, U.S.A.

Astronomitscheskij Institut, Sternberga, Moscow, Russia.

Observatoire, Beograd, Yugoslavia.

W. T. Hay, Hendon, London, N.W. 4, England.

\section{Actual List of Subscribers to circulars only}

Institute for Theoretical Astrophysics, Blindern (Oslo), Norway.

Observatoire astronomique, Jassy, Roumania.

National Observatory of the Czechoslovak Republic, Ondfejov, Czechoslovakia.

Lick Observatory, Mt Hamilton, Calif., U.S.A.

Yerkes Observatory, Williams Bay, Wis., U.S.A.

Mt Wilson Observatory, Pasadena, Calif., U.S.A.

U.S. Naval Observatory, Washington, D.C., U.S.A.

Students' Observatory, Berkeley, Calif., U.S.A.

Observatoire astronomique, Cracow, Poland.

Observatoire, Lyon, St Genis Laval, France.

Issei Yamamoto, Kyoto, Japan.

Société astronom, tcheque, Observatoire Stefanik, Prague-Petřín, Czechoslovakia.

Dominion Observatory, Kelburn, Wellington, New Zealand.

Observatoriet, Lund, Sweden.

Astrophysikal. Observatorium, Stará Ďala, Czechoslovakia.

Obserwatorium Astronomiczne, Warszawa, Poland.

Brown University Library, Providence, R.I., U.S.A.

Observatoire de Juvisy, Seine et Oise, France.

James Stokley, The Franklin Institute, Philadelphia, Pa., U.S.A.

Institut Astronomique de l'Université Charles, Prague-Smíchov, Czechoslovakia.

Osservatorio astronomico della R. Università, Bologna, Italy.

Astronomisches Institut der Universität, Lwów, Poland.

Taschkent Astronom. Observatorium, Taschkent, Russia.

Sterrewacht, Lembang, Java.

Observatory of the University of Michigan, Ann Arbor, Michigan, U.S.A.

J. F. Cox, Laboratoire d'Astronomie de l'Université, Bruxelles, Belgium.

The Union Astronomer, Union Observatory, Johannesburg, South Africa.

Observatoire de Toulouse, Toulouse, France.

Mount Lubomir Observatory, p. Kasina Wielka, Wojef. Cracow, Poland.

Axel Corlin, Lund, Sweden.

Institut d'Astrophysique de l'Université de Liége, Cointe-Sclessin, Belgium.

H. P. Hollis, Blackheath, London, S.E. 3, England.

Oswald Thomas, Wien III, Austria.

Institut d'Astronomie Pratique, Warszawa, Poland.

H.M. Nautical Almanac Office, London, S.E. Io, England.

Hans Q. Rasmusen, Værslevgaard, Værslev, Denmark.

Runde Taarns Observatorium, Copenhagen, Denmark.

Solar Physics Observatory, Cambridge, England.

Dunsink Observatory, Dublin, Ireland. 
Ole Römer-Observatoriet, Aarhus, Denmark.

G. F. Kellaway, Yeovil, England.

Nizamiah Observatory, Hyderabad-Deccan, India.

Glavnaja Astronomiceskaja Observatorija, Pulkovo, Leningrad, Russia.

E. M. Lindsay, Armagh Observatory, Northern Ireland.

H. Dubosq, Poitiers, France.)

R. Henseling, Berlin-Frohnau, Germany.

The Astronomical Society of Edinburgh, Edinburgh, Scotland.

ELIS STRÖMGREN

February 26, 1938 